\title{
Accepting Blame for Safe Tunneled Exceptions
}

\author{
Yizhou Zhang* Guido Salvaneschi ${ }^{\dagger} \quad$ Quinn Beightol* $^{*}$ \\ Barbara Liskov ${ }^{\ddagger} \quad$ Andrew C. Myers* \\ ${ }^{*}$ Cornell University, USA $\quad{ }^{\dagger}$ TU Darmstadt, Germany $\quad{ }^{\ddagger}$ MIT, USA \\ yizhou@cs.cornell.edu salvaneschi@cs.tu-darmstadt.de qeb2@cornell.edu \\ liskov@csail.mit.edu andru@cs.cornell.edu
}

\begin{abstract}
Unhandled exceptions crash programs, so a compile-time check that exceptions are handled should in principle make software more reliable. But designers of some recent languages have argued that the benefits of statically checked exceptions are not worth the costs. We introduce a new statically checked exception mechanism that addresses the problems with existing checked-exception mechanisms. In particular, it interacts well with higher-order functions and other design patterns. The key insight is that whether an exception should be treated as a "checked" exception is not a property of its type but rather of the context in which the exception propagates. Statically checked exceptions can "tunnel" through code that is oblivious to their presence, but the type system nevertheless checks that these exceptions are handled. Further, exceptions can be tunneled without being accidentally caught, by expanding the space of exception identifiers to identify the exception-handling context. The resulting mechanism is expressive and syntactically light, and can be implemented efficiently. We demonstrate the expressiveness of the mechanism using significant codebases and evaluate its performance. We have implemented this new exception mechanism as part of the new Genus programming language, but the mechanism could equally well be applied to other programming languages.
\end{abstract}

Categories and Subject Descriptors $\quad$ D.3.3 [Programming Languages]: Language Constructs and Features-Control structures

Keywords Exception tunneling; exception handling; Genus

Permission to make digital or hard copies of all or part of this work for personal or classroom use is granted without fee provided that copies are not made or distributed for profit or commercial advantage and that copies bear this notice and the full citation on the first page. Copyrights for components of this work owned by others than ACM must be honored. Abstracting with credit is permitted. To copy otherwise, or republish, to post on servers or to redistribute to lists, requires prior specific permission and/or a fee. Request permissions from Permissions@acm.org.

PLDI'16, June 13-17, 2016, Santa Barbara, CA, USA

(c) 2016 ACM. 978-1-4503-4261-2/16/06...\$15.00

http://dx.doi.org/10.1145/2908080.2908086

\section{Introduction}

Exceptions make code more reliable by helping programmers handle abnormal or unusual run-time conditions. The core idea is to transfer control in a nonlocal way to handler code that can be factored out from common-case code. This separation of concerns simplifies code and prompts programmers not to forget about exceptional conditions.

There has been disagreement since the 1970's about how or whether exceptions should be subject to static checking $[19,28]$. This disagreement continues to the present day [17]. Some currently popular languages_Java [20] and Swift [46] - offer checked exceptions that the compiler statically ensures are handled. However, exceptions are not part of type checking in other popular languages such as C++ [44], C\# [23], Scala [36], and Haskell [38].

Proponents of static checking argue that exceptions represent corner cases that are easy to forget. The evidence suggests they have a point. One study of a corpus of C\# code [8] determined that $90 \%$ of the possible exceptions are undocumented. Undocumented exceptions make it hard to know whether all exceptions are handled, and these unhandled exceptions percolate up through abstraction layers, causing unexpected software failures. Statically checked exceptions help programmers build more robust code [48].

Opponents of static checking argue that the annotation burden of statically checked exceptions does not pay offthat statically checked exceptions are too rigid to support common design patterns and common ways in which software evolves $[11,51]$. They, too, have a point. The problems with statically checked exceptions have become more apparent in recent years as object-oriented (OO) languages like C\#, Scala, and Java have acquired lambda expressions and the use of higher-order functions has become more common. As a result, the promise of exceptions to help make software more reliable has been partly lost.

Studies of the effectiveness of exception mechanisms have concluded that existing mechanisms do not satisfy the appropriate design criteria $[6,7]$. C\# does not statically check exceptions because its designers did not know how to design such an exception mechanism well, saying "more thinking is needed before we put some kind of checked exceptions 
mechanism in place" [22]. It seems the long-running conflict between checked and unchecked exceptions can be resolved only by a new exception mechanism.

This paper aims to provide a better exception mechanism, one that combines the benefits of static checking with the flexibility of unchecked exceptions. The new mechanism gives programmers static, compile-time guidance to ensure exceptions are handled, but works well with higher-order functions and design patterns. It adds little programmer burden and even reduces that burden. The run-time overhead of the mechanism is low, because exception handling does not require stack-trace collection in common use cases and avoids the need to wrap checked exceptions inside unchecked ones.

Two main insights underlie the new design. The first is that the distinction between "checked" and "unchecked" should not be a property of the type of the exception being raised, as it is in Java, but rather a property of the context in which the exception propagates. In contexts that are aware of an exception, the exception should be checked statically to ensure that it is handled. To handle higher-order functions and design patterns, however, some contexts must be oblivious to exceptions propagating through them; exceptions should tunnel uncaught through oblivious contexts, effectively unchecked.

This principle implies that the same exception may be both checked and unchecked at different points during its propagation. To prevent oblivious code from accidentally catching exceptions, a second insight is needed: exceptions can be distinguished by expanding the space of exception identifiers with an additional label that describes the exception-aware context in which this exception can be caught. These labels can be viewed as an extension of the notion of blame labels found in previous work on gradual typing [50]. Unlike with gradual typing, this sort of "blame" is not a programmer error; it is instead a way to indicate that exceptions should tunnel through the oblivious code until they arrive at the right exception-aware context.

We start the rest of the paper by exploring requirements for a good exception mechanism in Section 2. Sections 3-6 present our new exception mechanism informally in the context of a Java-like language. Section 7 defines a core language whose semantics show more precisely how the mechanism works. Using this core language, we prove the key theorem that all exceptions are handled explicitly. Section 8 describes our implementation of the new exception mechanism in the context of the Genus programming language [53]. The effectiveness of the mechanism is evaluated in Section 9, using code drawn from real-world codebases. Related work is explored more fully in Section 10; we conclude in Section 11.

\section{Design Principles for Exceptions}

The goal of an exception mechanism is to simplify and regularize the handling of exceptional events, making programs more reliable. However, there are two quite different classes of exceptional events, with different design goals:

- Failures. Some events cannot reasonably be expected to be handled correctly by the program-especially, events that arise because of programmer mistakes. Other events such as running out of memory also fall into this category.

- Unusual conditions. Other events arise during correct functioning of the program, in response to an unusual but planned-for state of the environment, or even just an unusual case of an algorithm.

These two classes place different requirements on the exception mechanism. For failures, efficiency is not a concern because the program is not expected to recover. However, programmers need the ability to debug the (stopped) program to discover why the failure occurred, so it is important to collect a stack trace. Furthermore, since failures imply violation of programmer assumptions, having to declare them as part of method signatures or write handler code for them is undesirable. Nonetheless, there are cases where the ability to catch failure exceptions is useful, such as when building frameworks for executing code that might fail.

For the second class of exceptions, unusual conditions, the design goals are different. Now efficiency matters! Because exceptions are slow in many common languages, programmers have learned to avoid using them. One insight is that because unusual conditions are part of the correct functioning of the program, the overhead of collecting a stack trace is unnecessary.

Unfortunately, existing languages tend not to support the distinction between these two exception classes well. For example, typical Java usage always leads to stack-trace collection, making exceptions very expensive. At the same time, code is cluttered with handlers for impossible exceptions.

\subsection{Higher-Order Functions and Exceptions}

Current exception mechanisms do not work well in code that uses higher-order functions. An example is an ML-style map method that applies a function argument to each element of a list, returning a new list. Callers of the higher-order function may wish to provide as an argument a function that produces exceptions to report an unusual condition that was encountered, such as an I/O exception. Of course, we want these exceptions to be handled. But the implementation of map knows nothing about these exceptions, so if such exceptions do occur, they should be handled by the caller of map. It is unreasonable for map either to handle or to declare them-its code should be oblivious to the exceptions.

This example illustrates why otherwise statically typed functional programming languages such as ML and Haskell do not try to type-check exceptions statically [25]. The problem has also become more prominent in modern OO languages that have added lambda expressions and are increasingly relying on libraries that use them (e.g., JavaFX, Apache Spark). The problem is encountered even without lambda ex- 
pressions, though; for example, Java's Iterator interface declares no exceptions on its methods, which means that implementations of Iterator are not allowed to generate exceptions-unless they are made "unchecked".

Our goal is to achieve the notational convenience of the functional programming languages along with the assurance that exceptions are handled, which is offered by languages such as Java, Swift [46], and Modula-3 [34]. We propose that a higher-order function like map should be implementable in a way that is oblivious to the exceptions possibly generated by its argument. The possible exceptions of the argument should not be declared in the signature of map; nor should the code of map have to say anything about these exceptions.

A subtle problem arises when a higher-order function like map uses exceptions inside its own implementation. If the exceptions of the argument and the internal exceptions collide, the map code could then accidentally catch exceptions that are not intended for it-an effect we call exception capture by analogy with the problem of variable capture in dynamically scoped languages [43]. For modularity, a way is needed to tunnel such exceptions through the intermediate calling context. In fact, accidentally-caught exceptions are a real source of serious bugs $[10,13]$.

An alternative to our oblivious-code approach that has been suggested previously $[18,24,41,47]$ is to parameterize higher-order code like map over the unknown exceptions of its argument. This exception polymorphism approach requires writing annotations on oblivious code yet still permits accidental exception capture.

\subsection{Our Approach}

Backed by common sense and some empirical evidence, we believe that code is more reliable when compile-time checking guides programmers to handle exceptional cases. It is disappointing that recent language designs such as C\# and Scala have backed away from statically declared exceptions.

We propose a new statically checked exception mechanism that addresses the weaknesses of prior exception mechanisms:

- It supports static, local reasoning about exceptions. Local reasoning is efficient, but more importantly, it aids programmer understanding. A code context is required to handle only the exceptions it knows about statically.

- The mechanism is cheap when it needs to be: when exceptions are used for nonlocal control flow rather than failures.

- In the failure case, however, the mechanism collects the stack trace needed for debugging.

- It supports higher-order functions whose arguments are other functions that might throw exceptions to which the higher-order code is oblivious.

- It avoids the exception-capture problem both for higherorder functions and for failures.

\section{The Exception Mechanism}

We use Java as a starting point for our design because it is currently the most popular language with statically checked exceptions. Our design is presented as a version of the Genus language, a variant of Java with an expressive constrained genericity mechanism [53]. The essential ideas should apply equally well to other languages, such as Java, C\# [31], Scala [36], and ML [32]. Since exception-oblivious code (like map) is often generic, it is important to study how exceptions interact with sophisticated generics. ${ }^{1}$

Previous languages have either had entirely "checked" or "unchecked" exceptions (in Java's terminology), or, as in Java, have assigned exception types to one of these two categories. Our insight is that "checked" vs. "unchecked" is a property of the context of the exception rather than of its type. Any exception should be "checked" in a context that is not oblivious to the exception and can therefore handle it. But in a context that is oblivious to the exception, the exception should be treated as "unchecked".

Genus requires that a method handle or explicitly propagate all exceptions it knows can arise from operations it uses or methods it calls. If the implementation of a method explicitly throws an unhandled exception whose type (or the supertype thereof) is not listed in the method's header, the program is rejected.

Like in Java, exceptions can be handled using the trycatch construct, and a finally block that is always executed may be provided. (The try-with-resources statement of Java 7 is easily supported; it is orthogonal to the new features.)

A method that wishes merely to propagate an exception to its caller can simply place a throws clause in its signature. We say such an exception propagates in checked mode. Unlike in Java, exceptions in checked mode do not cause stack-trace collection.

\subsection{Failures}

Unlike Java, our mechanism makes it easy for the programmer to indicate that an exception should not happen. The programmer ordinarily does this by putting a fails clause in the method header. Any caller of the method is then oblivious to the exception, meaning that the exception will be treated as unchecked as it propagates further. When code fails because of an exception, the exception propagates in a special mode, the failure mode.

For example, a programmer who is certain that the object class can be loaded successfully can write

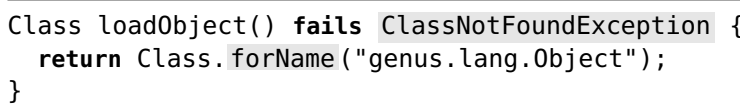

where the method forName in Class declares ClassNotFoundException in its throws clause. Note that a fails clause

\footnotetext{
${ }^{1}$ Genus uses square brackets rather than angle brackets for generic type arguments: List [T] rather than List $<\mathrm{T}>$. For brevity, we use Genus syntax and the Genus equivalents of Java core classes without further explanation.
} 
is really part of the implementation rather than part of the method signature or specification. We write it in the header just for convenience.

Exceptions propagating in failure mode also differ in what happens at run time. Programmers need detailed information to debug the stopped program to discover how the failure occurred. Therefore, failure exceptions collect a stack trace. This is relatively slow (as slow as most Java exceptions!) but efficiency is not a concern for failures.

\subsection{Avoiding Exception Capture}

Since exceptions propagating in failure mode do not appear in method signatures, it is important to avoid catching them accidentally. For example, consider the following code that calls two functions $\mathrm{g}(\mathrm{)})$ and $\mathrm{h}(\mathrm{)}$ :

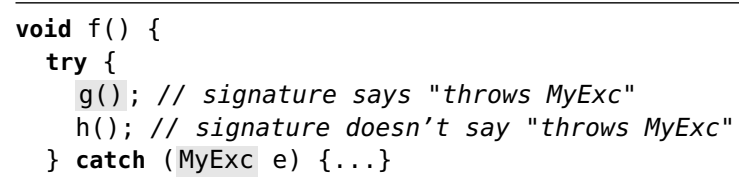

Suppose that because of a programmer mistake, the call to $\mathrm{h}$ ( ) unexpectedly fails with exception MyExc. If this exception were caught by the catch clause, $f()$ would execute code intended to compensate for something that happened in $g()$. We prevent this undesirable exception capture by ensuring that failure exceptions cannot be caught by any ordinary catch clauses: failure exceptions tunnel past all ordinary catch clauses.

Although exceptions in failure mode are not normally handled, there may be value in catching them at the top level of the program or at the boundary between components, to allow for more graceful exit. Genus supports this with a rarely used catch all construct that catches all exceptions of a given type, regardless of propagation mode. For example, if the try statement above were extended to include a second clause catch all (MyExc e) $\{\ldots\}$, the first catch clause would catch the expected MyExcs thrown by g, and the second catch all clause would catch failure-mode MyExcs tunneled through $\mathrm{h}$.

\subsection{Fail-by-Default Exceptions}

Java has a commonly accepted set of exceptions that usually correspond to programmer mistakes: the built-in subclasses of RuntimeException or Error. To reduce annotation burden for the programmer, our mechanism does not ordinarily require writing a fails clause in order to convert such exceptions to failure mode. We say these exceptions fail by default.

Fail-by-default exceptions are different from Java's unchecked exceptions. Unchecked exceptions conflate failures with ordinary exceptions that are tunneling through oblivious code but that still ought to be subject to static checking.

In contrast, fail-by-default exceptions remain in checked mode until they reach a method-call boundary; they convert from checked mode to failure mode only if the current method does not declare the exception in its throws clause.

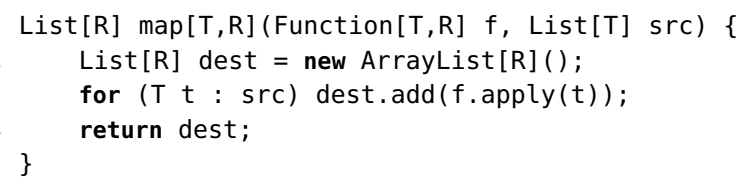

Figure 1: A higher-order function written in Genus

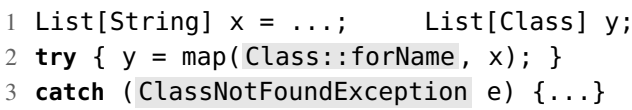

Figure 2: Passing a function that throws extra exceptions

A fail-by-default exception collects a stack trace only if and when it does fail, so code can still handle exceptions like NoSuchElementException efficiently. It is therefore reasonable and useful to write code that handles such exceptions.

Genus also permits the set of fail-by-default exceptions to be changed on a per-class basis by adding a throws or fails clause to the class definition.

\section{Higher-Order Abstractions and Tunneling}

As discussed in Section 2.1, higher-order functions pose a problem for statically checked exception mechanisms. The same problem arises for many common object-oriented design patterns, which are essentially higher-order functions. Our solution is to tunnel exceptions through oblivious code.

For example, Genus allows the programmer to pass to the higher-order function map (Figure 1) an implementation of Function that throws exceptions, even though the signature of Function does not mention any exceptions. If the passed Function throws an exception, that exception is tunneled through the exception-oblivious code of map to the caller of the exception-aware code that called map.

In the code of Figure 2, the method forName in class Class is passed to map. This call is legal even though forName is declared to throw an exception ClassNotFoundException. Since map is oblivious to this exception, it cannot be expected to handle it. By contrast, the caller of map is aware that an object that throws exceptions is used at a type (Function) that does not declare any exceptions. Because it is aware of the exception, the caller is responsible for the exception.

If ClassNotFoundException arises at run time, it tunnels through the code of map and is caught by the catch clause. Alternatively, the caller could have explicitly converted the exception to a failure (via a fails clause) or explicitly allowed it to propagate (via a throws clause). In any case, exception handling is enforced statically.

\subsection{Exception Tunneling is Safe and Light}

In Java, the rigidity of checked exceptions has led to some verbose and dangerous idioms, especially when higher-order functions and design patterns are used. Exception tunneling helps avoid these undesirable programming practices.

In particular, Java programmers often abandon static checking of exceptions to make it possible for their excep- 


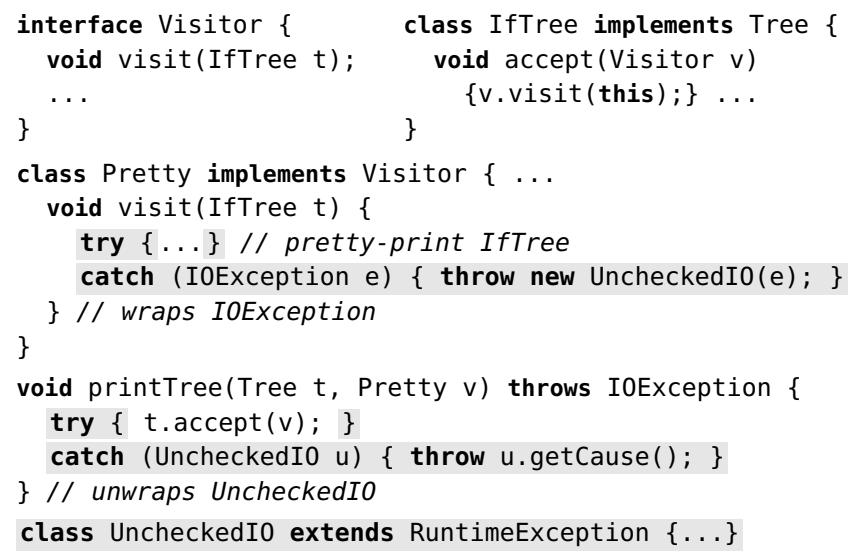

Figure 3: The pretty-printing visitor in javac (simplified). Code for exception wrapping and unwrapping is highlighted.

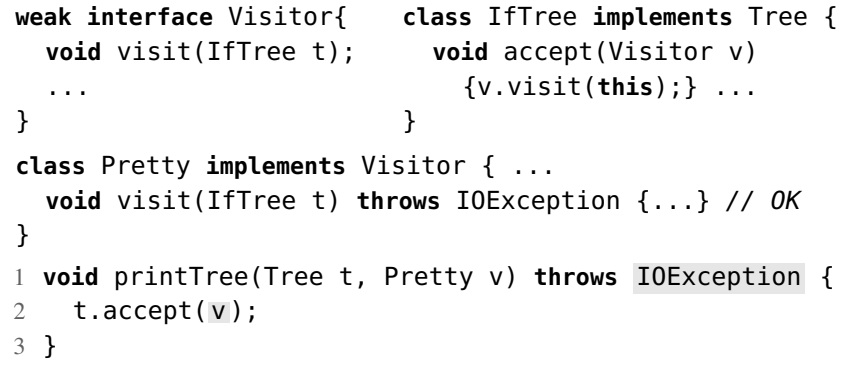

Figure 4: Exception tunneling in javac, ported to Genus.

tions to pass through exception-oblivious higher-order code. They either define their own exceptions as unchecked, or they cope with preexisting checked exceptions by calling unsafe APIs like sun.misc.Unsafe: : throwException [29], or by wrapping checked-exception objects inside uncheckedexception objects. Wrapping with unchecked exceptions is the safest of these workarounds because it makes exception capture less likely, but wrappers are verbose and expensive.

Figure 3 shows an example of this idiom taken from the javac compiler [37], which contains a number of visitors for the Java AST. In order to conform to the Visitor interface, the visit methods in the pretty-printing visitor (Pretty) wrap the checked IOException into unchecked wrappers, which are then unwrapped as shown in method printTree. This programming pattern is verbose, abandons static checking, and is likely to be slow due to stack-trace collection.

When written in Genus, the same visitor pattern (Figure 4) does not require exception wrapping or unwrapping to achieve tunneling. The modifier weak on the interface Visitor (see Section 4.4) makes it legal for its implementations to declare new exceptions (the interface Function is annotated similarly). In printTree, the call $t$.accept ( $v$ ) passes a visitor object that throws the additional exception IOException. If this exception is thrown by the visitor, it tunnels until it reaches printTree. Thus, the antipattern of exception wrapping becomes unnecessary.

\begin{tabular}{l|c|c|c} 
& Checked & Tunneling & Failure \\
\hline Local, static checking & Yes & No & No \\
Stack-trace collection & No & No & Yes
\end{tabular}

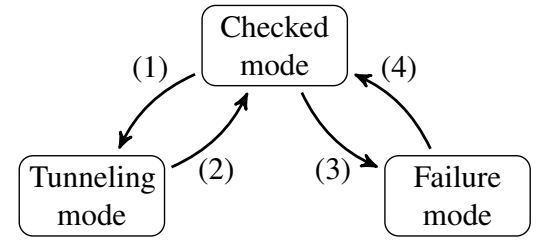

(1)(2): $\S 4.2$ and $\S 4.3 \quad$ (3)(4): $§ 3.1$ and $\S 3.3$

Figure 5: Three exception propagation modes

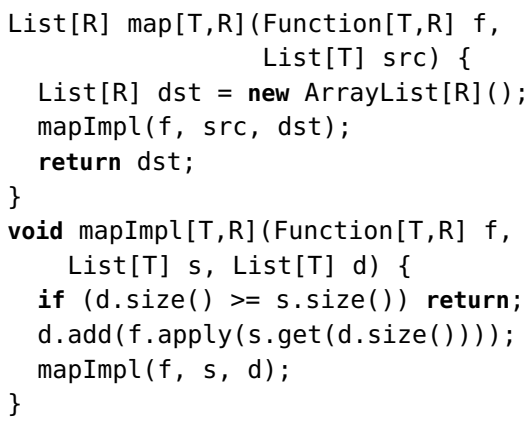

Figure 6: A recursive implementation of map (left) and a stack snapshot showing propagation of an exception caused by $f$ (right). The stack grows downwards.

\subsection{Tunneling Checked Exceptions}

Earlier we discussed two modes of exception propagation: checked mode and failure mode. Tunneling mode is a third mode of propagation. The relationships between the three modes are summarized in Figure 5. In tunneling mode, as in checked mode, static checking enforces handling of exceptions. As in failure mode, these exceptions do not need to be declared in signatures of the methods they tunnel through.

A given exception may propagate in more than one mode. Consider the alternative, slightly contrived implementation of map in Figure 6. It calls a helper method mapImpl, which then recursively calls itself to traverse the list. Suppose an exception arises when function $f$ is applied to the sixth element in the list. Figure 6 shows a snapshot of the current call stack. Since $f$ is the place where the exception is generated, the exception first propagates in checked mode within the function $f$. Because its caller mapImpl is oblivious to the exception, the exception then switches to tunneling mode and propagates through all the mapImpl stack frames. Finally, the caller of map knows about the exception and thus can handle it. The exception returns to checked mode when it reaches this caller. From there, it can be either caught, rethrown in checked mode, or turned into failure.

\subsection{Tunneling, Exception Capture, and Blame}

Tunneling avoids the phenomenon of exception capture discussed in Section 3. In OO languages like Java and C\#, exception capture occurs because of an unexpected collision in 


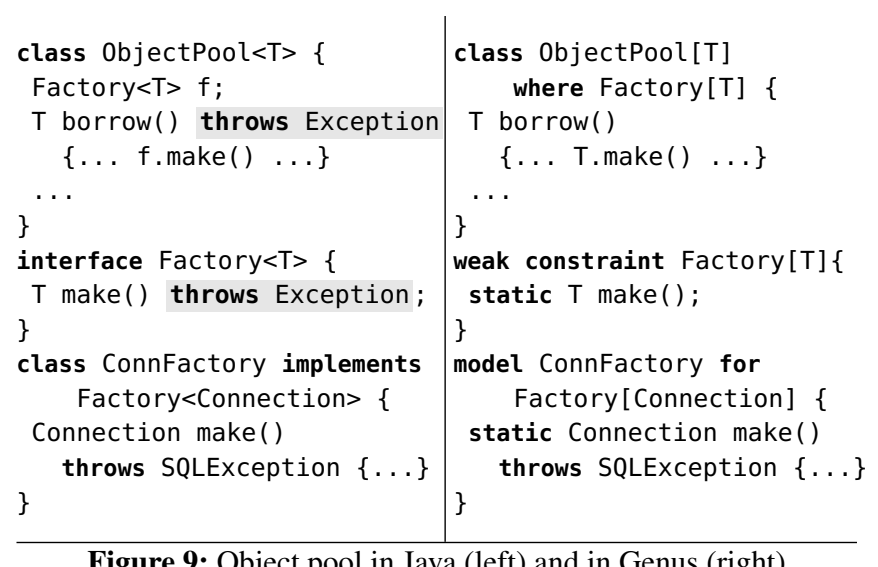

Figure 9: Object pool in Java (left) and in Genus (right)

fore, an overriding method cannot add new exceptions to the supertype's signature for the method.

Our mechanism relaxes this requirement for weak types. Methods of an inexact subtype must obey the supertype specification-except that they can throw more exceptions. This implies that their additional exceptional conditions must be signaled with different types than those in the supertype method-Tokenizer indicates an I/O problem by throwing IOException, not NoSuchElementException - and that the exceptional conditions the supertype defines must not be signaled in other ways-Tokenizer cannot issue a failure or return null when the iteration has no more elements.

\section{Generics and Exceptions}

We have also used Genus to explore the important interaction between exceptions and mechanisms for constrained parametric polymorphism. Various languages constrain generic type arguments in various ways: for example, Java and C\# use subtyping constraints, whereas Haskell and Genus use the more flexible mechanism of type classes [49].

Genus provides constrained parametric polymorphism via constraints and models [53]. Like type classes, Genus constraints are predicates describing type features required by generic code. Genus models show how types can satisfy constraints, like type class instances in Haskell. Unlike Haskell instances, models are explicitly part of the instantiation of a generic abstraction. For example, the two instantiations Set [String] and Set[String with CaseInsensEq] are different types distinguished by the use of the model CaseInsensEq in place of default string equality. This distinction is helpful for precisely reasoning about exceptions.

As with interfaces, we would like the flexibility to instantiate generic abstractions with types whose operations throw additional exceptions not provided for by the constraint. Thus, similar to interfaces, Genus constraints can be weak; models may be inexact with respect to the weak constraints they witness.

The example in Figure 9 shows the utility of this feature, comparing Java and Genus code for an object pool abstraction. The left side of the figure shows an example adapted from the Apache Commons project [2]. The abstract factory type Factory defines a method make with a signature that declares "throws Exception". This idiom is common in Java libraries, because it permits subtypes to refine the actual exceptions to be thrown.

However, such declarations are a source of frustration for Java programmers [51]. Consider that method make is called by the method borrow in objectPool to create a new object in case there is no idle one, so borrow must also declare "throws Exception". The precise exception (in the example, SQLException) is therefore lost. Clients of ObjectPool<Connection> must handle Exception, which is no better, and perhaps worse, than having an unchecked exception. Further, client code that handles the overly general exception is more likely to suffer from exception capture.

The right side of Figure 9 shows a reasonable way to implement the example in Genus. A constraint Factory [T] is used to express the requirement that objects of type $T$ can be made in some way; because it does not declare any exceptions, method borrow need not either. However, a model like ConnFactory can add its exceptions such as SQLException to the method make. Client code can then use the type ObjectPool[Connection with ConnFactory] to recycle Connection objects. Because the model is part of the type, it is statically apparent that the exception SQLException may be thrown; the client code will be required to handle this exception-but only this exception.

\section{Exactness Analysis}

The new exception mechanism poses new challenges for type checking. One challenge is that the identity of an exception includes a blame label, so blame should not be allowed to escape its scope. Otherwise, an exception might not be handled.

For example, consider the first definition of the Peeking Iterator class in Figure 10. It decorates a wrapped Iterator in field inner to support one-element lookahead. Its next and peek methods call inner. next ( ). Per Section 4.4, it is possible to pass an object of some inexact subtype of Iterator to the constructor, to be assigned to inner. However, Iterator is a weak type, so the methods of the actual object being passed may throw exceptions not declared by Iterator. If the assignment to inner were allowed, the same exceptions would propagate when the next method of the PeekingIterator object were called. And this call could be delayed until outside the context that is aware of the mismatch with Iterator. In this case, the exceptions would not be guaranteed to be handled.

Therefore, we want to detect statically that the assignment to inner lets the blame from the inexact object escape. Storing an inexact object into a data structure at a weak type, or even returning an inexact object from a method, may permit such an escape of blame.

A second challenge for the new exception mechanism, and indeed for exception mechanisms generally, is that pro- 


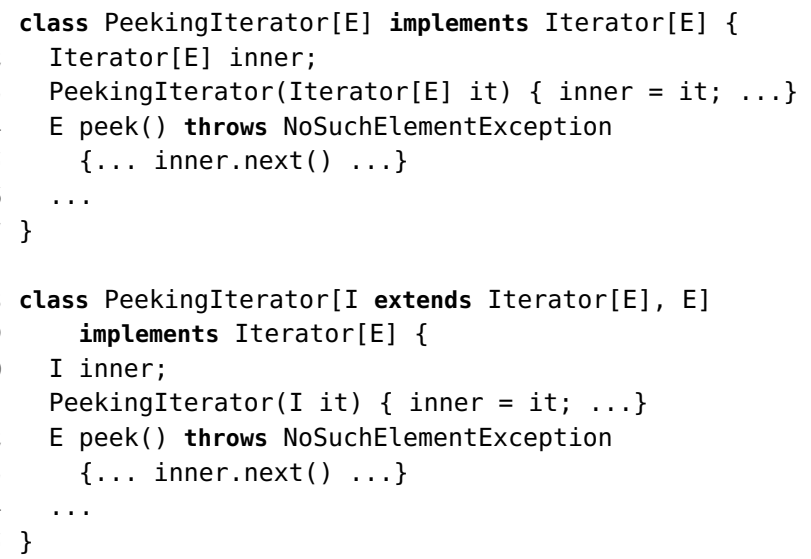

Figure 10: Two definitions of PeekingIterator. The top one allows blame to escape, but a warning is issued for the constructor. The bottom one uses dependent exactness to soundly avoid the warning.

grammers should not be forced to write handlers for program points where exceptions cannot happen. To address this challenge, the location of blame should be precise. Figure 8 offers an example of this problem. The method call iter. next() might appear (to the compiler) not to throw any exceptions because iter has type Iterator [Token], yet we know that it may throw an IOException because iter is initialized to a Tokenizer. A safe, conservative solution would be to require all of the code below this initialization to be wrapped in a try-catch. But this solution would make it difficult to continue the iteration after an IOException is raised.

Genus addresses these two challenges using an intraprocedural program analysis that assigns exactness to uses of weak types, with little annotation effort by the programmer.

\subsection{Exactness Annotations and Exactness Defaults} Indicating intolerance of inexactness at use sites. When a formal parameter or local variable is assigned a weak type, the programmer can annotate the type with @exact to indicate that an exact subtype must be used. For example, if the programmer adds @exact to the formal parameter of the constructor at line 3 in Figure 10,

PeekingIterator(@exact Iterator $[E]$ it) $\{$ inner $=i t ; \ldots\}$

it becomes a static error to pass an inexact implementation of Iterator to the constructor. So it is guaranteed that using inner will not generate unexpected exceptions. By contrast, without @exact, Genus issues a warning about the assignment to the escaping pointer inner at line 3 . If the exception is actually thrown at run time, it is converted into failure and the usual stack trace is collected.

Although @exact might appear to add notational burden, our experience with porting existing Java code into Genus (Section 9) suggests that this escape hatch is rarely needed. We have not seen the need to use the @exact annotation to dismiss such warnings in existing code ported from Java.
It seems that programmers use weak types differently from other types-weak types provide functionality rather than structure. Further, exactness defaults and exactness inference (Section 6.2) reduce annotation overhead, and exactnessdependent types (Section 6.3) provide more expressiveness.

Exactness defaults. Exactness defines what exceptions that are not declared by the weak type might nevertheless be generated by the term being typed. Exactness $\mathcal{E}$ is formally a mapping from methods to sets of exceptions. These mappings form a lattice ordered as follows:

$$
\mathcal{E}_{1} \leq \mathcal{E}_{2} \Leftrightarrow \operatorname{dom}\left(\mathcal{E}_{1}\right) \subseteq \operatorname{dom}\left(\mathcal{E}_{2}\right) \wedge \forall m . \mathcal{E}_{1}(m) \subseteq \mathcal{E}_{2}(m)
$$

The bottom lattice element is strict exactness, denoted by $\varnothing$.

To avoid the need for programmers to write the annotation @exact for most uses of weak types, the compiler determines exactness using a combination of exactness defaults and automatic exactness inference. To see how these mechanisms work, consider the code in Figure 11.

1. Weak types used as return types or field types are exact, as these are the channels through which pointers can escape. For these types, we have $\mathcal{E}=\varnothing$.

2. Methods and constructors are implicitly polymorphic with respect to exactness. That is, they can be viewed as parameterized by the exactness of their argument and receiver types.

3. Weak types in a local context are labeled by exactness variables: for example, $x$ and $y$ in Figure 11, at lines 2 and 11 respectively.

A unification-based inference engine solves for these variables, inferring exactness from the local variable uses (Section 6.2).

4. For a procedure call, an exactness variable is generated for each argument and/or receiver whose formal parameter type is weak. Exactness variables of this kind in Figure 11 are $z$ (line 9) and $w$ (line 12).

5. Recall that Genus supports constrained parametric polymorphism via type constraints [53]. Subtype constraints and where-clause constraints can also specify exactness. Their default exactness is deduced in ways similar to those listed above. For example, in Figure 11 the use of Runnable in g's signature (line 1) constrains the type parameter $\mathrm{T}$, so its exactness is resolved to a fresh name $e$, with respect to which $\mathrm{g}$ is polymorphic.

\subsection{Solving Exactness Constraints}

At each assignment (including variable initialization and argument passing) to a variable with weak type $\tau$, inexact values must not escape into variables with exact types. Therefore, constraints of form $\tau\left\langle\mathcal{E}_{r}\right\rangle \leq \tau\left\langle\mathcal{E}_{l}\right\rangle$ are generated, where $\mathcal{E}_{l}$ is the exactness of the left-hand side expected type $\tau$ in an assignment, resolved as described in Section 6.1, and $\mathcal{E}_{r}$ is the exactness of the right-hand side actual type with respect 


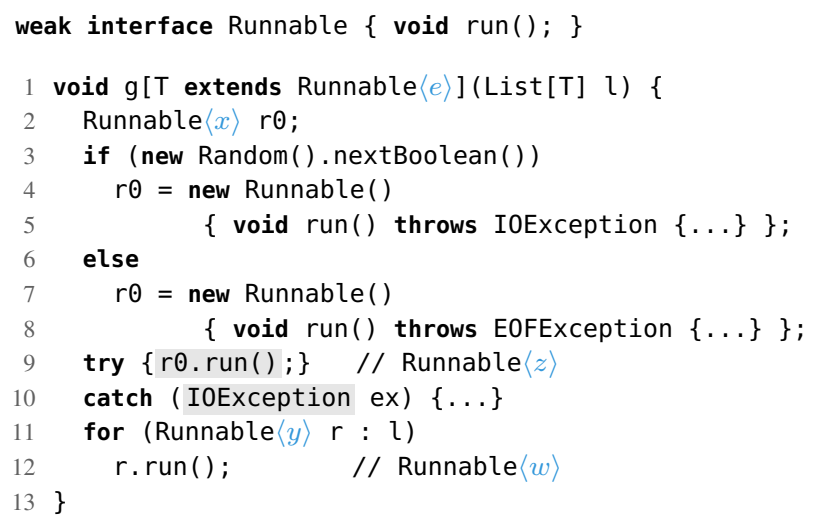

Figure 11: Running example for exactness analysis. Uses of weak types are tagged by exactness variables, to be fed into the solver. (EOFException is a subtype of IOException.)

to $\tau$. For example, the code of Figure 11 then generates the following constraints with line numbers attached:

$$
\begin{array}{rlr}
\tau\left\langle\mathcal{E}_{r}\right\rangle & \leq \tau\left\langle\mathcal{E}_{l}\right\rangle & \\
\text { Runnable }\langle\{\text { run } \mapsto \text { IOException }\}\rangle & \leq \operatorname{Runnable}\langle x\rangle & 4 \\
\text { Runnable }\langle\{\text { run } \mapsto \text { EOFException }\}\rangle & \leq \operatorname{Runnable}\langle x\rangle & 7 \\
\text { Runnable }\langle x\rangle & \leq \operatorname{Runnable}\langle z\rangle & 9 \\
\text { Runnable }\langle e\rangle & \leq \operatorname{Runnable}\langle y\rangle & 11 \\
\text { Runnable }\langle y\rangle & \leq \operatorname{Runnable}\langle w\rangle & 12 \\
\text { Iterator[T] }\langle\varnothing\rangle & \leq \operatorname{Iterator}[\mathrm{T}]\langle v\rangle & 11
\end{array}
$$

The last constraint is due to desugaring of the loop at lines 11-12:

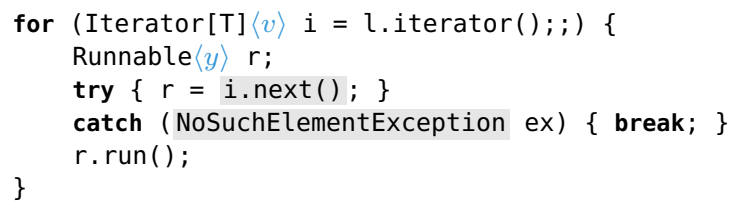

Note that enhanced for loops are translated differently by Java and Genus. Genus exceptions are fast enough that it is often faster to call next ( ) and catch a final exception, rather than calling hasNext ( ) on every iteration.

The compiler solves these constraints by finding the least upper bounds of $x, y, z, w$, and $v$ :

$$
\begin{aligned}
& x=z=\{\text { run } \mapsto\{\text { IOException, EOFException }\}\} \\
& y=w=e \\
& v=\varnothing
\end{aligned}
$$

The fact that a solution exists implies that inexact pointers will not escape to the heap, addressing the first challenge of escaping blame.

The solution also reveals precisely where exception handling is required, addressing the second challenge of blame precision. Specifically, the solution to each variable generated via the fourth case of exactness defaults (Section 6.1) tells what exception can be produced by the method call. In our running example, the solution to $z$ is that the method call rO. run () may throw IOException or EOFException, which are handled by the catch block. Notice that although mis- matches happen at lines 4 and 7, the blame does not take effect until the method call at line 9 .

The solution to $w$ is the polymorphic label $e$, which means that the current context is oblivious to whatever exceptions correspond to $e$ when $\mathrm{g}$ is called, as discussed in Section 4.

\subsection{Exactness-Dependent Types}

It is possible to write a type-safe PeekingIterator using the @exact annotation, but we might want a peeking iterator that throws the same exceptions as its underlying iterator does. This expressiveness can be obtained by making PeekingIterator talk about the potential mismatch, as in the bottom definition of PeekingIterator in Figure 10. The class is now parameterized by the type of the iterator it decorates. In the using code below, PeekingIterator is instantiated on an inexact subtype of Iterator, so the compiler requires the exception to be handled:

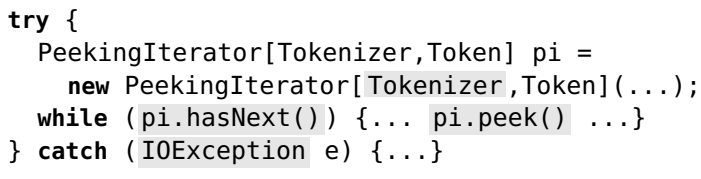

Parameterized by a weak constraint, the Genus version of objectPool in Figure 9 is similarly exactness-dependent.

The special this keyword, when referring to the current object of a weak class, also has an exactness-dependent type-it is dependent on the exactness of the run-time class of the object with respect to the enclosing weak class. Since the run-time class is statically unknown, the compiler must assume that it can add arbitrary exceptions. Thus it results in a compiler warning to use this in ways that generate blame. However, we expect this to be rare: most weak types are interfaces, which do not normally use this.

\section{Formalization}

We formalize the new exception mechanism using a core calculus, CBC (for Checked Blame Calculus).

\subsection{Syntax and Notations}

Figure 12 defines both a surface and a kernel syntax for CBC. Programs are written in the surface syntax, and rewritten to and evaluated in the kernel calculus. Applications in surface terms are tagged by unique blame labels (ranged over by $\ell$ ) representing lexical positions where blame can arise. Rewriting propagates these labels from the surface language to the kernel language, for blame tracking during kernel evaluation.

The surface syntax assumes a fixed set of exception names ranged over by $E$. The kernel syntax allows them to be labeled to form new names; $E$ and $E^{\ell}$ are different names.

Surface types $(\tau)$ include weak types and strong types. A strong type $(\sigma)$ is either the base type $B$ or a function type $\tau \stackrel{\perp}{\rightarrow}[\sigma]_{\bar{E}}$ that does not allow mismatch against $\bar{E}$. (An overline denotes a (possibly empty) set.) A weak type $\tau \stackrel{\top}{\rightarrow}[\sigma]_{\bar{E}}$, on the other hand, tolerates mismatch. Notice that function return types must be strong to prevent blame from 


\begin{tabular}{|c|c|c|c|}
\hline Surface terms & $f$ & $::=$ & $\begin{array}{l}b|x| \lambda x: \tau . f\left|\left\{f_{1} f_{2}\right\}^{\ell}\right| \\
\text { let } x: \tau \leftarrow f_{1} \text { in } f_{2} \mid \\
\text { throw } E \mid \text { try } f_{1} \text { catch } E \triangleright f_{2} \\
\sigma \mid \tau \stackrel{\tau}{\longrightarrow}[\sigma]_{\bar{F}}\end{array}$ \\
\hline Surface strong types & $\sigma$ & $::=$ & $B \mid \tau \stackrel{\perp}{\longrightarrow}[\sigma]_{\bar{E}}$ \\
\hline Kernel terms & $e$ & $::=$ & $\begin{array}{l}b|x| \lambda x: T . e\left|e_{1} e_{2}\right| \\
\text { let } x: T \leftarrow e_{1} \text { in } e_{2} \mid \\
\text { throw } U \mid \text { try } e_{1} \text { catch } \overline{U \triangleright e_{2}}\end{array}$ \\
\hline Kernel types & $T$ & $::=$ & $S \mid T \stackrel{\top}{\longrightarrow}[S]_{\bar{U}}$ \\
\hline Kernel strong types & $S$ & $::=$ & $B \mid T \stackrel{\perp}{\longrightarrow}[S]_{\bar{U}}$ \\
\hline Kernel exceptions & $U$ & $::=$ & $E \mid E^{\ell}$ \\
\hline Environments & $\Gamma$ & $::=$ & $\varnothing \mid \Gamma, x: \tau\langle\bar{E}\rangle$ \\
\hline Escape kind & $K$ & $::=$ & $\top \mid \perp$ \\
\hline $\begin{array}{l}\mathrm{K}(\sigma)=\perp \\
\mathrm{K}\left(\tau \stackrel{\mathrm{T}}{\longrightarrow}[\sigma]_{\bar{E}}\right)=\end{array}$ & & & $\begin{array}{l}\Gamma_{\perp}=\{x: \sigma\langle\varnothing\rangle \mid x: \sigma\langle\varnothing\rangle \in \Gamma\} \\
\Gamma_{\top}=\Gamma\end{array}$ \\
\hline
\end{tabular}

Figure 12: Syntax of CBC

escaping. The same can be said about kernel types. Since there is an obvious injection (syntactic identity) from surface types to kernel types, we abuse notation by using $\tau$ and $\sigma$ in places where kernel types $T$ and $S$ are expected.

Environments $\Gamma$ contain mappings from variables to their types and exactness. Exactness is represented by sets of exceptions. Strong types enforce strict exactness, so their exactness is represented by $\varnothing$. The auxiliary function $\mathrm{K}(\cdot)$ returns $\perp$ for strong types and $T$ for weak types; values of strong types must not escape. $\Gamma_{\perp}$ retains only the strongly-typed variables in $\Gamma$.

\subsection{Semantics}

Surface-to-kernel rewriting. Figure 13 defines the rewriting rules. The judgment $\Gamma ; K \vdash f \rightsquigarrow e:[\tau]_{E}$ translates the surface term $f$ to the kernel term $e$, assigns $f$ the type $\tau$, and infers the exceptions $\bar{E}$ that evaluating $e$ might raise.

Typing is dependent on $K$, which indicates whether the term in question is guaranteed not to escape. For example, the left-hand-side term in an application is type-checked with $K=\top$ as in R-APP-E and R-APP-I, while the body of an abstraction is type-checked with $K=\perp$ as in R-ABS. RVAR-W denies first-class citizenship to weakly typed variables, and augments the return type if the environment indicates inexactness.

Exception mismatch is computed using the subtyping relation $\lesssim_{E}$, as in R-APP-I and R-LET. But only with R-APP-I can blame take effect, and its translation is consequently less obvious. To avoid exception capture, we need to give new names to exceptions involved in the mismatch. Therefore, the translation wraps the argument in an abstraction, which, when applied, catches any exceptions in the mismatch $(\bar{E})$ and rethrows their labeled versions $\left(\overline{E^{\ell}}\right)$. The caller is aware of the exceptions $\bar{E}$, so it catches the labeled exceptions tunneled to its context and strips off their labels.
Semantics of the kernel calculus. The static and dynamic semantics of the kernel are omitted here for lack of space but can be found in the technical report [54]. The static semantics is largely similar to the typing induced by rewriting, except that the kernel need not worry about exception capture. Hence, exception propagation happens in the usual way.

\subsection{Type Safety}

The type system guarantees that if a program can be typed without exceptional effects, it cannot get stuck when evaluated, or terminate in an exception. This guarantee easily follows from two other standard results.

First of all, the kernel type system is sound:

\section{Theorem 1 (Kernel soundness: preservation and progress)}

$$
\begin{aligned}
& \text { - If } \varnothing ; K \vdash e:[T]_{\bar{U}} \text { and } e \longrightarrow e^{\prime} \text { then } \varnothing ; K \vdash e^{\prime}:[T]_{\bar{U}} \text {. } \\
& \text { - If } \varnothing ; K \vdash e:[T]_{\bar{U}} \text { then either } \\
& \text { 1. } \exists v \cdot e=v \text {, or } \\
& \text { 2. } \exists U_{0} \in \bar{U} \cdot e=\text { throw } U_{0} \text {, or } \\
& \text { 3. } \exists e^{\prime} . e \longrightarrow e^{\prime} .
\end{aligned}
$$

Proof. This is proved in the usual way, by induction on the kernel typing derivation. See the technical report for details of this proof and of other formal results in this paper.

Second, the translation from the surface language to the kernel language is type-preserving:

\section{Lemma 1 (Rewriting preserves types)}

If $\Gamma ; K \vdash f \rightsquigarrow e:[\tau]_{\bar{E}}$ then $\Gamma ; K \vdash e:[\tau]_{\bar{E}}$.

Proof. By induction on the derivation of the translation.

The guarantee that well-typed programs handle their exceptions is a direct corollary of these two previous results:

\section{Corollary 1 (No uncaught exceptions)}

If $\varnothing ; \perp \vdash f \rightsquigarrow e:[\tau]_{\varnothing}$ then $\exists v . e \longrightarrow^{*} v$.

\section{Implementation}

We have implemented the new exception mechanism for the Genus programming language. The implementation consists of about 5,800 lines of code, extending the compiler for the base Genus language [53]. Genus is implemented using Polyglot [35], so code generation works by translating to Java code, using a Java compiler as a back end.

The rest of this section focuses on the translation into Java. The translation is guided by two goals: 1) it should prevent accidental capturing of exceptions, and 2) it should add negligible performance overhead to normal control flow.

\subsection{Representing Non-Checked Exceptions}

Unlike exceptions in checked mode, exceptions traveling in tunneling mode or failure mode must acquire new identities to avoid accidental capturing. These identities are implemented by wrapping exceptions into objects of classes Blame and Failure. Both classes extend RuntimeException, but Blame does not collect a stack trace. 


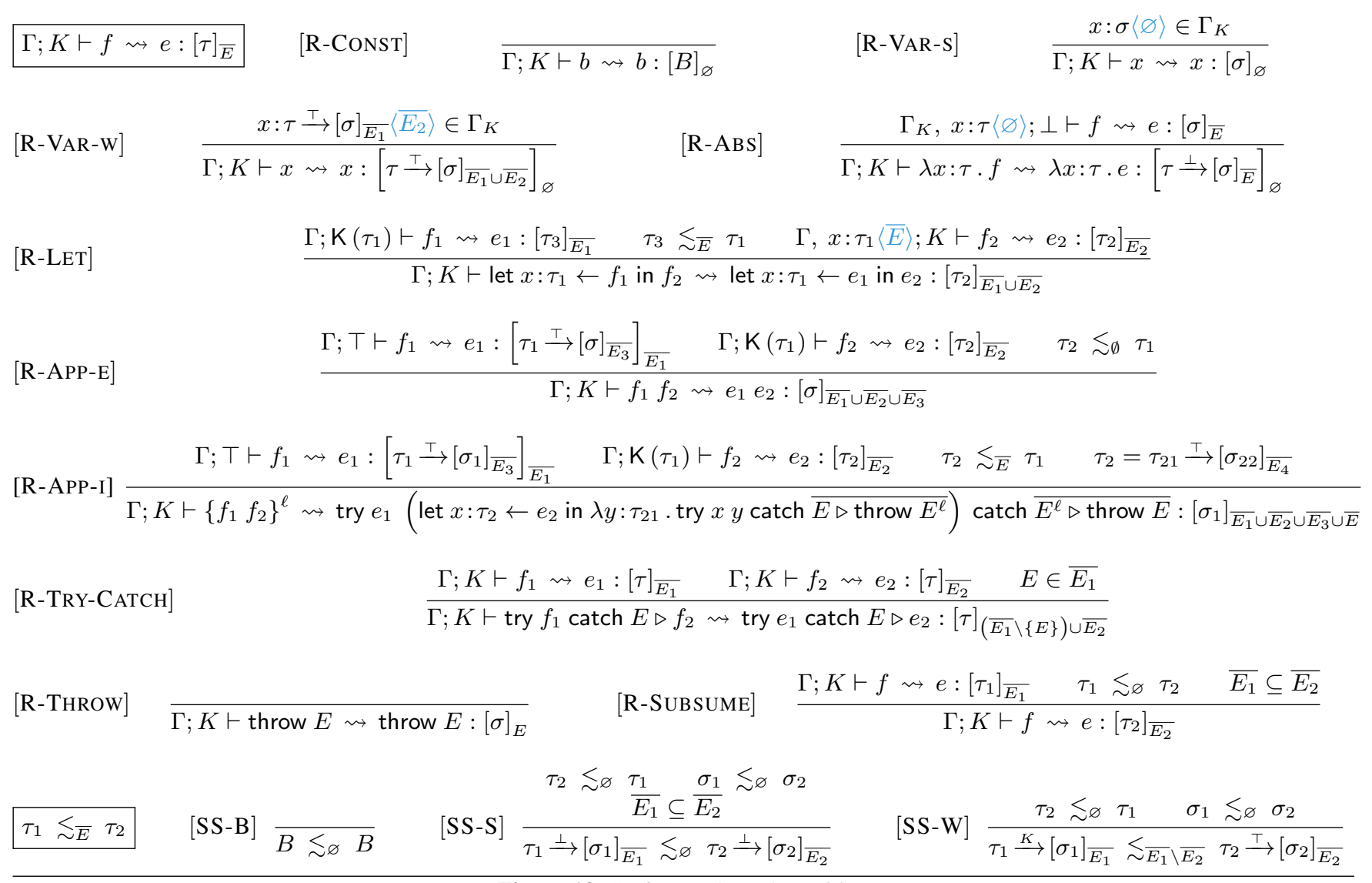

Figure 13: Surface-to-kernel rewriting

\subsection{Translating Exception-Oblivious Code}

Methods with weakly typed parameters ignore extra exceptions generated by them. To ensure they are handled in the appropriate context, weakly typed parameters, including the receiver, are accompanied by an additional Blame argument that serves as the blame label. When the actual argument is exact, the Blame argument is null. For example, the weak type Iterator has the following translation:

interface Iterator $<\mathrm{E}>\{$

E next\$Iterator(Blame $b \$$ ) throws NoSuchElementException; ...

\}

It receives a $\mathrm{Blame}$ object from its caller to accompany the weakly typed receiver. If an implementation of Iterator throws a mismatched exception, it is wrapped in this Blame object and tunneled through the code oblivious to it.

Exception-oblivious procedures are translated in a similar way. For example, the code generated for map (Figure 1) looks like the following:

$<T, R>$ List $<R>\operatorname{map}($ Function $<T, R>f$, List $<T>$ src, Blame $b \$$ ) $\{\ldots$ f.apply\$Function $(t, b \$) \ldots\}$

The extra Blame argument $b \$$ is intended for potential mismatch in the argument function $f$, and is passed down to the method call $f$.apply\$Function (...) so that exceptions from $f$ have the right blame label.

\subsection{Translating Exception-Aware Code}

The definition of Iterator's inexact subtype Tokenizer is exception-aware. Its translation is shown in the left of Figure 14. Per Java's type-checking rules, the overriding method in Tokenizer cannot throw extra checked exceptions. Instead, the overriding method next\$Iterator(Blame) redirects to method next() that does the real work, possibly throwing an I0Exception, and then turns that exception into either a Blame or a Failure, which is unchecked. If the Blame argument is not null, there must be a program point ready to handle the exception. So the IOException is wrapped in the Blame object and is tunneled to that program point. If the Blame object is null, a Failure object wrapping the I0Exception is created and thrown. This might happen, for example, if the programmer chose to disregard the compiler warning reported for passing a Tokenizer (Figure 7) into the constructor of PeekingIterator (top of Figure 10).

The code in Figure 8 is also exception-aware, and Figure 14 (right) shows its translation. Instead of creating a new Blame object every time a mismatch happens, each thread maintains a Blame object pool that recycles Blame objects. A $\mathrm{Blame}$ object is borrowed from the pool at the blamable pro- 


\begin{tabular}{c|c|c}
\hline Java w/ stack & Genus & Java w/o stack \\
\hline 7.19 & 1.16 & 1.16
\end{tabular}

Table 1: Performance with EasyI0 (s)

\begin{tabular}{c|c|c}
\hline mechanism & $\begin{array}{c}\text { exception } \\
\text { objects }\end{array}$ & time (ns) \\
\hline \multirow{2}{*}{ Java exceptions } & new instance & 817.7 \\
\cline { 2 - 3 } & cached & 124.7 \\
\hline Java unchecked wrappers & cached & 826.0 \\
\hline $\begin{array}{c}\text { Genus exceptions } \\
\text { (tunneled) }\end{array}$ & new instance & 139.8 \\
\cline { 2 - 3 } & cached & 128.6 \\
\hline
\end{tabular}

Table 2: Exception tunneling microbenchmarks

Figure 15 reports the results of this comparison. Each reported measurement averages at least 20 runs, with a standard error less than $1.2 \%$. Benchmark parameters were set so that each run took more than 30 seconds.

Overall, the extended compiler generates slightly faster code than the original compiler. The average speedup is $2.4 \%$, though performance varies by benchmark. The speedup is caused largely by the exception-based translation of enhanced for loops.

Performance of exception-heavy code. To evaluate the performance improvement that Genus obtains by avoiding stack-trace collection on realistic code, we measured the running times of pattern-matching regular expressions using the EasyI0 package. It makes heavy use of exceptions to control search. The running times are shown in Table 1. Each number averages 10 runs, with $6.2 \mathrm{M}$ exceptions thrown in each run.

Stack-trace collection in Java causes more than $6 \times$ overhead compared to Genus exceptions. Genus targets Java, so it is not surprising that similar performance can be achieved with Java, if stack-trace collection is turned off and exception objects are cached.

Exception tunneling performance. We used a microbenchmark to explore the overhead of exception tunneling, the key difference between Genus and Java. The microbenchmark performs method calls on objects passed down from a higherorder function; the method call either throws an exception or returns immediately, and is prevented from being inlined. Since there is no other work done by the method, performance differences are magnified.

The results are shown in Table 2. We compare exception tunneling in Genus to two (unsafe) Java workarounds: typical Java exceptions and unchecked-exception wrappers. We also refine the comparison for typical Java exceptions and tunneled Genus exceptions based on whether a new exception object is created for each throw; throwing a single cached instance is reasonable for non-failure exceptions carrying no extra information. Each number averages 20 runs, with a standard error less than $0.6 \%$ of the mean.

The rightmost column measures time to exit via an exception. Genus exceptions perform well because they do not collect a stack trace. The slowdown compared to the second row is mostly because exception mismatch requires borrow and handback calls (Section 8.3) in every loop iteration.

On the other hand, Genus exceptions significantly outperform unchecked wrappers, the safest way to tunnel in Java. Java's performance is poor here because an uncheckedexception object is created for each raised exception, whereas the implementation of Genus recycles Blame objects.

The microbenchmark also measures the time to return from a method normally. The average cost of a call and return in Java was $6.0 \mathrm{~ns}$. In the absence of mismatch, our translation adds the overhead of passing a null pointer to the normal return path, increasing the cost slightly to $6.3 \mathrm{~ns}$. The results in Figure 15 suggest this increase is negligible in practice.

\section{Related Work}

Notable approaches to exceptions. PL/I was the first language with exceptions. It supports user-defined exceptions with exception handlers dynamically bound to exceptions [28]. Goodenough [19] and Liskov and Snyder [26] introduced statically scoped handlers. CLU [26] was the first language to support some static checking of exceptions. Exceptions are declared in function signatures, and thrown exceptions must appear in these signatures. If not explicitly resignaled, propagating exceptions automatically convert to failure. Mesa [33] supports both termination- and resumption-style exceptions but does not check them statically. Ada [1] attaches handlers to blocks, procedures, or packages. Unhandled exceptions propagate automatically, but exceptions are not declared. Eiffel [30] exceptions originate from the violation of assertions and are raised implicitly. Upon exceptions, programmers can retry the execution with different parameters; otherwise, exceptions implicitly propagate to callers. Modula-3 [34] introduced fully statically checked exceptions. Black [3] and Garcia et al. [16] present comparative studies of some exception mechanisms.

Empirical findings. An empirical study by Cabral and Marques [7] shows that in Java and .NET exception handlers are not specialized enough to allow effective handling, which we believe is partly attributable to a lack of documentation of exceptions in the .NET case [8] and the rigidity of checked exceptions in the Java case. Robillard and Murphy [40] identify the global reasoning required of programmers as a major reason why exceptions are hard to use.

Exception analysis. Function types in functional languages such as ML and Haskell do not include exceptions because they would interfere with the use of higher-order functions. Exception capture can be avoided in SML [32] because exception types are generative, but other variants of ML lack this feature. Leroy and Pessaux [25] observe that uncaught exceptions are the most common failure mode of large ML applications, motivating them and others [12] to develop program analyses to infer exceptions. Such analyses can be helpful, especially for usage studies [52], but they necessarily involve trading off performance and precision, and entail non- 
local reasoning that does not aid programmers in reasoning about their code. A benefit of our mechanism is that it is likely to lead to more accurate, scalable static analyses, because precise exceptions largely remove the need to approximate exceptional control flow [5, 42].

Exception polymorphism. Some recent designs attempt to address the rigidity of checked exceptions through exception polymorphism: anchored exceptions [47] as a Java extension, polymorphic effects [41] as a Scala extension, rowpolymorphic effect types in the Koka language [24], and the rethrows clause introduced in Swift 2 [46]. These approaches add annotation burden to exception-oblivious code; more fundamentally, they do not address exception capture.

Blame tracking. Our notion of blame is related to that introduced by work on contracts [14] and further developed by work on gradual typing [50], in which blame indicates where fault lies in the event of a contract violation (or cast failure). In our setting, an exception mismatch results in static blame being assigned that indicates where "fault" lies should an exception arise at run time, with the compiler statically checking that the "faulty" program point handles exceptions.

Blame has polarity $[14,50]$; in our setting, exception mismatch at covariant (or contravariant) positions gives rise to positive (or negative) blame. Existing mechanisms for checked exceptions only consider positive blame; exceptions with negative blame are the missing piece. By contrast, in Genus both kinds of exceptions are subject to static checking, and our implementation and formalization manifest their difference: exceptions with negative blame acquire new identities to achieve safe tunneling.

Type inference. Our exactness inference bears resemblance to work on gradual type inference [39]. Both inference algorithms encode escape analysis "for free", although the motivation for escape analysis differs. Our approach to default exactness (Section 6.1) is similar to default region annotations in a region-based type system [21].

\section{Conclusions}

Our new exception mechanism combines the benefits of static checking with the flexibility of unchecked exceptions. We were guided in the design of this mechanism by thinking carefully about the goals of an exception mechanism, by much previous work, and by many discussions found online. Our formal results and experience suggest that our approach improves assurance that exceptions are handled. The evaluation shows that the mechanism works well on real code. It adds negligible cost when exceptions are not being used; exception tunneling comes with a small performance penalty that appears to be more than offset in practice by avoiding the run-time overhead of wrapping exceptions. We hope this work helps programmers use exceptions in a principled way and gives language implementers an incentive to make exceptions more efficient.

\section{Acknowledgments}

We thank Owen Arden, Chinawat Isradisaikul, Jed Liu, Michael Hicks, and the anonymous reviewers for discussions about exception mechanisms and for suggestions about this paper. This research has been supported by MURI grant FA9550-12-1-0400, administered by the US Air Force, by NSF grant 1513797, and by the European Research Council, grant No. 321217. This paper does not necessarily reflect the views of any of its sponsors.

\section{References}

[1] Ada 95. Ada 95 reference manual: language and standard libraries, 1997.

[2] Apache Commons. The Apache Commons project. https: //commons . apache. org/.

[3] A. P. Black. Exception handling: The case against. $\mathrm{PhD}$ thesis, University of Oxford, 1982.

[4] S. M. Blackburn, R. Garner, C. Hoffmann, A. M. Khang, K. S. McKinley, R. Bentzur, A. Diwan, D. Feinberg, D. Frampton, S. Z. Guyer, M. Hirzel, A. Hosking, M. Jump, H. Lee, J. E. B. Moss, A. Phansalkar, D. Stefanović, T. VanDrunen, D. von Dincklage, and B. Wiedermann. The DaCapo benchmarks: Java benchmarking development and analysis. In $21^{s t}$ ACM SIGPLAN Conf. on Object-Oriented Programming, Systems, Languages and Applications (OOPSLA), pages 169-190, 2006.

[5] M. Bravenboer and Y. Smaragdakis. Exception analysis and points-to analysis: Better together. In Proceedings of the Eighteenth International Symposium on Software Testing and Analysis, ISSTA '09, 2009.

[6] P. A. Buhr and W. Y. R. Mok. Advanced exception handling mechanisms. IEEE Trans. Softw. Eng., 26(9), Sept. 2000.

[7] B. Cabral and P. Marques. Exception handling: A field study in Java and .NET. In $21^{\text {st }}$ European Conf. on Object-Oriented Programming, pages 151-175, 2007.

[8] B. Cabral and P. Marques. Hidden truth behind .NET's exception handling today. IET Software, 1(6), 2007.

[9] B. Cahoon and K. S. McKinley. Data flow analysis for software prefetching linked data structures in Java. In International Conference on Parallel Architectures and Compilation Techniques (PACT), Sept. 2001.

[10] CWE. Common weakness enumeration list. http://cwe. mitre.org/data/.

[11] B. Eckel. Does Java need checked exceptions? http://www. mindview. net/Etc/Discussions/CheckedExceptions, 2003.

[12] M. Fähndrich, J. S. Foster, A. Aiken, and J. Cu. Tracking down exceptions in standard ML programs. Technical report, EECS Department, UC Berkeley, 1998.

[13] FindBugs bug descriptions. Findbugs bug descriptions. http: //findbugs. sourceforge. net/bugDescriptions.html/.

[14] R. B. Findler and M. Felleisen. Contracts for higher-order functions. In $7^{\text {th }}$ ACM SIGPLAN Int'l Conf. on Functional Programming, 2002.

[15] R. B. Findler, M. Latendresse, and M. Felleisen. Behavioral contracts and behavioral subtyping. In ESEC/FSE-9, 2001. 
[16] A. F. Garcia, C. M. Rubira, A. Romanovsky, and J. Xu. A comparative study of exception handling mechanisms for building dependable object-oriented software. Journal of Systems and Software, 2001.

[17] B. Goetz. Java theory and practice: The exceptions debate. http://www.ibm.com/developerworks/library/ j- jtp05254, 2004.

[18] B. Goetz. Exception transparency in Java. http: //blogs.oracle.com/briangoetz/entry/exception transparency_in_java, 2010.

[19] J. B. Goodenough. Exception handling: Issues and a proposed notation. Comm. of the ACM, 18:683-696, Dec. 1975.

[20] J. Gosling, B. Joy, G. Steele, and G. Bracha. The Java Language Specification. Addison Wesley, 3rd edition, 2005. ISBN 0321246780.

[21] D. Grossman, G. Morrisett, T. Jim, M. Hicks, Y. Wang, and J. Cheney. Region-based memory management in Cyclone. In ACM SIGPLAN Conf. on Programming Language Design and Implementation (PLDI), pages 282-293, 2002.

[22] A. Hejlsberg, B. Venners, and B. Eckel. Remaining neutral on checked exceptions. http://www.artima.com/intv/ handcuffs. html, 2003.

[23] A. Hejlsberg, S. Wiltamuth, and P. Golde. The C\# Programming Language. Addison-Wesley, 1st edition, Oct. 2003. ISBN 0321154916.

[24] D. Leijen. Koka: Programming with row polymorphic effect types. In 5th Workshop on Mathematically Structured Functional Programming, 2014.

[25] X. Leroy and F. Pessaux. Type-based analysis of uncaught exceptions. ACM Trans. on Programming Languages and Systems, 22(2), Mar. 2000.

[26] B. Liskov and A. Snyder. Exception handling in CLU. IEEE Transactions on Software Engineering, SE-5(6):546-558, Nov. 1979.

[27] B. H. Liskov and J. M. Wing. A behavioral notion of subtyping. ACM Trans. on Programming Languages and Systems, 16(6), Nov. 1994.

[28] M. D. MacLaren. Exception handling in PL/I. In Proceedings of an ACM Conference on Language Design for Reliable Software, 1977.

[29] L. Mastrangelo, L. Ponzanelli, A. Mocci, M. Lanza, M. Hauswirth, and N. Nystrom. Use at your own risk: The Java Unsafe API in the wild. In 2015 ACM SIGPLAN Conf. on Object-Oriented Programming, Systems, Languages and Applications (OOPSLA), 2015.

[30] B. Meyer. Eiffel: The Language. 1992.

[31] Microsoft Corporation. Microsoft C\# Language Specifications. Microsoft Press, 2001. ISBN 0-7356-1448-2.

[32] R. Milner, M. Tofte, and R. Harper. The Definition of Standard ML. MIT Press, Cambridge, MA, 1990.

[33] J. G. Mitchell, W. Maybury, and R. Sweet. Mesa language manual. Technical Report CSL-78-1, Xerox Research Center, Palo Alto, CA, Feb. 1978.

[34] G. Nelson, editor. Systems Programming with Modula-3. Prentice-Hall, 1991.

[35] N. Nystrom, M. R. Clarkson, and A. C. Myers. Polyglot: An extensible compiler framework for Java. In $12^{\text {th }}$ Int'l Conf. on
Compiler Construction (CC'03), pages 138-152, Apr. 2003.

[36] M. Odersky. The Scala Language Specification. EPFL, 2014. Version 2.9.

[37] OpenJDK javac. The javac compiler. http://hg.openjdk. java.net/.

[38] S. Peyton Jones. Haskell 98 language and libraries: the revised report. Cambridge University Press, 2003.

[39] A. Rastogi, A. Chaudhuri, and B. Hosmer. The ins and outs of gradual type inference. In $39^{\text {th }}$ ACM Symp. on Principles of Programming Languages (POPL), 2012.

[40] M. P. Robillard and G. C. Murphy. Designing robust Java programs with exceptions. In Proceedings of the 8th ACM SIGSOFT International Symposium on Foundations of Software Engineering (FSE-8), 2000.

[41] L. Rytz, M. Odersky, and P. Haller. Lightweight polymorphic effects. In $26^{\text {th }}$ European Conf. on Object-Oriented Programming, 2012.

[42] S. Sinha and M. J. Harrold. Analysis and testing of programs with exception handling constructs. IEEE Transactions on Software Engineering, 26(9), Sept. 2000.

[43] G. L. Steele, Jr. Common LISP: the Language. Digital Press, second edition, 1990. ISBN 1-55558-041-6.

[44] B. Stroustrup. The C++ Programming Language. AddisonWesley, 1987.

[45] SunFlow. SunFlow: the open-source rendering engine. Opensource software, 2007.

[46] Swift 2014. Swift programming language. https:// developer . apple.com/swift/resources, 2014.

[47] M. van Dooren and E. Steegmans. Combining the robustness of checked exceptions with the flexibility of unchecked exceptions using anchored exception declarations. In $20^{\text {th }} A C M S I G$ PLAN Conf. on Object-Oriented Programming, Systems, Languages and Applications (OOPSLA), 2005.

[48] B. Venners. Failure and exceptions: A conversation with James Gosling, Part II. http://www.artima. com/intv/solid.html, 2003.

[49] P. Wadler and S. Blott. How to make ad-hoc polymorphism less ad hoc. In $16^{\text {th }}$ ACM Symp. on Principles of Programming Languages (POPL), 1989.

[50] P. Wadler and R. B. Findler. Well-typed programs can't be blamed. In European Symposium on Programming, 2009.

[51] R. Waldhoff. Java's checked exceptions were a mistake. http://radio-weblogs. com/0122027/stories/2003/ 04/01/JavasCheckedExceptionsWereAMistake. html, 2003.

[52] W. Weimer and G. C. Necula. Exceptional situations and program reliability. ACM Trans. on Programming Languages and Systems, 30(2), Mar. 2008.

[53] Y. Zhang, M. C. Loring, G. Salvaneschi, B. Liskov, and A. C. Myers. Lightweight, flexible object-oriented generics. In $36^{\text {th }}$ ACM SIGPLAN Conf. on Programming Language Design and Implementation (PLDI), pages 436-445, June 2015.

[54] Y. Zhang, G. Salvaneschi, Q. Beightol, B. Liskov, and A. C. Myers. Accepting blame: Expressive checked exceptions. Technical Report http://hdl . handle. net/1813/43784, Cornell University Computing and Information Science, Apr. 2016. 